2 TITLE: The Open Field Test

3 AUTHORS: Vootele Voikar and S. Clare Stanford

5 Vootele Voikar (corresponding author; e-mail: vootele.voikar@helsinki.fi; ORCID 0000-0003-4201-8666)

6 Neuroscience Center / Laboratory Animal Center

7 Helsinki Institute of Life Science (HiLIFE)

8 University of Helsinki

9 Finland

11 S. Clare Stanford (e-mail: c.stanford@ucl.ac.uk; ORCID 0000-0002-5623-1751)

12 Department of Neuroscience, Physiology and Pharmacology

13 University College London,

14 London WC1E 6BT

15 UK

\title{
17 Summary/Abstract
}

18 The Open Field Test (OFT) is the most widely used method for observing the behaviour of mice and rats 19 under laboratory conditions. It is used to evaluate the exploratory behaviour, general locomotor activity 20 and emotionality of rats and mice. However, it has to be kept in mind that these behaviours are not 21 independent, they interact and so a change in one will affect another. Despite being a seemingly simple 22 test, several caveats need to be acknowledged when selecting the variables for analysis and interpreting the data with regard to the aforementioned domains. The observed behaviours can depend on a number of procedural, environmental and biological factors, which should be carefully considered when planning the experiment, as well as during the analysis and interpretation of the results. This review provides a critical overview of these factors, followed by some warnings and practical tips for conducting the OFT.

\section{Key Words}

open field, mouse, rat, anxiety, exploration, emotionality, ambulation, locomotor activity

This is a pre-print of the following chapter: Vootele Voikar \& S. Clare Stanford, The Open field Test, published in Psychiatric Vulnerability, Mood, and Anxiety Disorders: Tests and Models in Mice and Rats, edited by Jaanus Harro, 2023, Humana New York, NY reproduced with permission of Humana New York, 


\section{INTRODUCTION}

\subsection{BACKGROUND AND SHORT HISTORICAL OVERVIEW}

The Open Field Test (OFT) is the most commonly used method for evaluating the spontaneous locomotor activity, emotionality and exploratory behaviour of rats and mice in a novel environment. The history of the OFT dates back to 1930s when Calvin Hall reported this method in a series of papers [1-3]. In Hall's experiments, the rat was exposed to a large, brightly-lit circular arena for two minutes, during which time the animal's ambulation was scored and fecal pellets counted. The test was repeated over several consecutive days and these measures were interpreted as an index of the animals' emotionality, although what aspect of emotionality was being evaluated was not specified.

Since then, the OFT and other 'novel environments' have been used extensively for studying emotionality and/or ambulatory activity of rats and mice. A comprehensive set of experiments was conducted by Peter L. Broadhurst for standardizing and further understanding of rat behaviour in the open field [4-6]. He highlighted the importance of environmental variables, genetic differences and the pre-test history of animals. In 1954, Broadhurst started a selective breeding program, which resulted in the generation of the Maudsley Reactive and Nonreactive rat strains. The strain designations (Reactive and Nonreactive) were related to their defecation scores in the OFT [7]. In addition to studies of emotionality, these strains have been investigated extensively for many other behavioural domains and their underlying physiology [8, 9].

Since the 1950s, several decades of research have provided a wealth of material that have invigorated debates about what aspects of animals' behaviour and emotionality are actually evaluated in the OFT [1013] and the number of publications that have reported using the "Open Field Test" has grown steadily, year on year (Figure 1). This increased output, particularly over the last 25 years, largely reflects the use of the OFT in experiments aimed at characterizing the phenotype of genetically-modified mice and as a high throughput screen for the effects of pharmacological compounds [14]. In such studies, the test is often used to evaluate either spontaneous locomotor activity or emotional behaviour [15-17]. However, that is problematic because locomotor activity and emotional behaviour are not independent variables, and a change in one of these aspects of behaviour will inevitably affect the other (see below).

In her book, "What's wrong with my mouse", Jacqueline Crawley strongly recommended a close collaboration with experts in behavioural neuroscience for carrying out behavioural phenotyping of knockout and genetically-modified mice [18]. Despite that warning, concerns have been expressed about potential "misuse" of the test, particularly through oversimplification and misinterpretation of the results, 
which arises from ignoring important fundamental factors that have been identified over decades of research [19-22]. Moreover, it has been argued even more strongly that "widely used assays such as the open-field test are performed at the wrong time, for inadequate durations and using inappropriate mouse 4 strains" [23].

\subsection{ETHOGRAM IN THE OPEN FIELD}

When studying changes in animals' behaviour following an experimental challenge (such as drug administration, neuronal lesion or genetic alteration), an important first step is to be confident about what counts as normal (baseline) behaviour of the test species in the context of the experiment. That rests on the assembly of a profile of the full behavioural repertoire of the animal (ethogram) - not simply focusing on the single aspect of behaviour that is of particular interest.

As with many other behavioural tests, the OFT was initially developed and validated for the rat. Yet, as has often been commented before, mice are not little rats. Indeed, there are important differences between the two species [24] and many of these differences depend on the test procedure, the environment and the behavioural parameters that are measured $[25,26]$. Unfortunately, the richness of species-specific behaviour is often ignored, but consideration of the animals' full behavioural repertoire is essential if the aim of the experiment is to draw conclusions about its emotional status, locomotor activity, or exploratory motivation $[27,28]$. In short, extreme caution is needed when adjusting any procedure that was developed using rats for studies of the mouse. Regardless of any differences in the innate behaviour of mice and rats, the basic procedure(s) for measuring exploratory and approach-avoidance (anxiety-like) behaviour in the OFT and other novel arenas are basically similar for both species $[29,30]$.

Tests for exploratory activity can be broadly divided into two main categories [31]. The forced exploration paradigm is the more common and involves exposing the animal to the novel arena, from the beginning of the experiment, without any possibility of escape. By contrast, in a "free exploration" protocol, the animal has the option to enter the arena from its home cage, or other safe and familiar "container", which is attached to, or placed within, the test arena. Some experimenters combine the OFT with object exploration tests, by placing an unfamiliar object in the centre of the arena and measuring the latency of the animal to approach the novel object and the time spent investigating it. Whichever approach is adopted, the duration of the OFT is highly variable - from 2-5 minutes up to 60-90 minutes. Yet, animals typically stop moving around the open field after about $20 \mathrm{~min}$. However, there are exceptions: some strains exhibit specific behavioural profiles, which become evident with prolonged 
exposure. For instance, the Balb/c mice show high avoidance in exploring the large open field arena during the initial 30 minutes of the test, followed by increased activity, by comparison with first-generation, captive wild (feral) mice [23, 32].

Longer periods of testing are usually applied when measuring drug effects (e.g., sedation, arousal) but, again, different mouse or rat strains exhibit different response profiles [33-35]. A longer duration of single exposure, or repeated testing, are usually interpreted as leading to habituation to the novel arena: i.e., it is assumed that the environment becomes familiar and no longer provokes exploration. However, there is rarely confirmation of that assumption, which must be assessed empirically, such as by monitoring changes in plasma corticosteroid or catecholamine concentrations. It should be borne in mind that, without that information, a reduction in exploration of the arena could indicate sensitization (quasi-freezing) rather than habituation.

Paradoxically, the major trap for measuring and interpreting the behaviour of a mouse or rat in the OFT lies in the perceived simplicity of the test. The most commonly reported parameters in the OFT are locomotor activity (ambulation, measured as total distance travelled) and time spent in the centre of the arena. Avoidance of the centre and increased time spent close to the wall (thigmotaxis) are used as an index of 'anxiety-like' behaviour [36]. This is despite evidence that drugs with established anti-anxiety effects in humans have inconsistent effects on rodent behaviour in the OFT, especially in respect of their activity in the centre of the field (e.g. [37]. This inconsistency was underlined by a comprehensive review, which revealed that only $56 \%$ of published studies (i.e., barely more than chance) reported an increase in animals' activity in the centre of the field after treatment with antianxiety drug [14]. These reports call into question the validity of the assumption that an increase in centre-field activity (or a reduction in thigmotaxis) reflects a reduction in animals' anxiety status.

In connection with this, a key point for interpretation of the behavioural measures in the OFT is the problem that locomotor activity and emotionality are not independent variables: a change in one will affect the other. This leads to the problem that centre-field activity will depend on animals' total activity in the OFT. The key parameter is the proportion of the animals' activity that is directed towards the centre of the field [38], which is often not taken into account. Similarly, some aspects of the animals' behaviour can be mutually exclusive: for instance, animals cannot move around the arena while grooming, scratching or rearing. This confound can have important consequences because some experimental challenges induce changes in some aspects of animals' behaviour, but not others $[39,40]$. 
1 Another factor to consider is that ambulation in the environment is not always exploratory, whereas other

2 behaviours (e.g., sniffing and rearing) can certainly involve an exploratory component, even though the

3 animal is not moving around the arena. Also, both mice and rats can establish a home-base in novel

4 environment: a specific location from where the exploratory trips or excursions are made into the remaining environment $[41,42]$.

Many of these problems can be ameliorated by measuring multiple ethological parameters, to build up a full profile of the animals' behaviour in the OFT. Detailed attention to the recorded pattern of all the animals' movements in the novel arena (not just ambulation) can greatly inform inferences about the structure and interetation of exploratory behaviour. This profile would include behaviours such as: rearing

11 [43-46], grooming [47-52], sniffing, risk-assessment (stretched attend postures and flat-back approach), tail rattling, freezing and jumping - all of which are interpreted as providing information on animal behaviour their emotional states, exploratory activity and motivation, curiosity and fear [53,54].

Observing and recording a comprehensive ethogram of animal's behaviour with score-sheets is the most important and valuable approach for its characterization [55-57]. However, manual scoring of behaviour is time-consuming, labour-intensive work, which is prone to errors and bias. With the increasing number of experiments, there has been progressively more pressure to automate the process through video-recording and computerized evaluation of the behaviour(s). Even so, errors in detection can arise due to improper calibration of the equipment for animal size or light conditions (reviewed in [58]), for example, and so the raw data and videos should be always checked for the quality of recording.

A current limitation of automated recording is that it is not ideal for counting multiple ethological parameters. To date, the behavioural parameters that are scored are typically restricted to the distance travelled by the animal and time spent in different zones of the arena. This precludes the possibility of taking into account other aspects of the animals' behaviour that could confound interpretation of the results (as explained above).

A major breakthrough in recent years has been the development of artificial intelligence and deep learning methods as promising solutions for improved scoring of behaviour expressed by animals in test situations [59-63]. Machine learning approaches bring enormous potential for automated, precise and unbiased scoring (notwithstanding the limitation that the (supervised) learning requires annotation of behaviours by

33 a human) or the exploration and detection of novel behavioural sequences and patterns (unsupervised 34 learning). The technology is growing and developing rapidly, but it is not without challenges. Indeed, the 35 large number of recorded variables definitely complicates the analysis and interpretation of the data. Large 
amounts of complex data will require advanced expertise in the dedicated areas of data-mining. For an interesting example of a research project that involves gathering extensive data on the spontaneous behaviour of group-housed mice in the home cage, see [64] and https://www.zooniverse.org/projects/rdot-bains/rodent-little-brother-secret-lives-of-mice/about/research. The successful development of this approach will also depend on sharing the codes and algorithms, validation and standardization of behavioural annotations (for different strains, test environments etc.), followed by uptake of these novel and complex methodologies by behavioural neuroscientists. These challenges are discussed comprehensively in [62].

In short, novel methods and advances in the technology for recording animal behaviour are most welcome,

11 but there is much work to do in terms of developing the meaningful ethological analysis that is needed for valid interpretation of the data-sets. Moreover, these should incorporate not only fine movements that involve specific body-points, but also complex behaviours that are affected by animals' motor function, exploratory motivation and emotional status.

\subsection{BIOLOGICAL FACTORS - SEX AND GENETIC BACKGROUND}

For a long time, it has been common practice to use only male rodents for behavioural experiments because of the belief that the estrous cycle in females increases the variability of their behaviour $[65,66]$. This view was reinforced by many studies that consistently demonstrated sex differences in the behaviour of male and female rats in the open field test (and other behavioural tests), with females being more active than males $[4,67-69]$.

However, it appears that the sex differences in mice are not so robust and may depend on many different factors (including strain, age, test conditions etc.) [33, 70-72]. Importantly, several recent studies, including meta-analysis of published reports, cast doubt on the view that the behaviour of females is more variable than that of males, [73-77]. These findings do not confirm that concerns about sex differences in rats were unjustified. Rather, they provide a strong justification for using both sexes in basic research as a matter of routine, especially considering the fact that the prevalence of many human disorders show clear sex differences too $[78,79]$. Whatever the case, the National Institutes of Health (USA) and other funding agencies now expect the sex of the animals to be a variable that should be factored into the research design, analyses and reporting of all experiments [80, 81].

34 Genetic background of the experimental animals is another biological determinant affecting the observed phenotype. In principle, laboratory rodents are divided into outbred stocks (heterogeneous populations) 
and inbred (isogenic) strains. It is interesting to note that the majority of rats used in biomedical research are regarded as outbred, whereas mice are mostly used as inbred strains. Also, there have been differences in standard practice that relate to specific scientific disciplines: e.g., in psychology and toxicology research, the use of outbred, heterogeneous animals has predominated.

There is no single or straightforward answer to the question of which genetic background should be used. Rather, the research question or hypothesis must inform the decision on which is the most suitable species and strain. Recent studies, which provide some evidence that outbred stocks are not necessarily more phenotypically variable than inbred strains [82], have added a further complicating factor. Surely, inbred strains provide a stable system for studying the effects of single genes (in the case of genetic engineering) or endophenotypes, or other experimental manipulations. For generalization of the outcome (external validation), which is essential for successful translation into humans, it is suggested that the experiment should include more than one inbred strain in the design [83, 84], or use an outbred strain. If the panel of inbred strains is used for measuring any trait, the strain distribution can be revealed $[85,86]$. However, knowing the typical characteristics of each strain is needed for determining possible ceiling or floor effects [87] which could confound the interpretation of the results.

\subsection{ENVIRONMENTAL AND PROCEDURAL FACTORS}

The list of variables that can affect the behaviour of animals in an open field and other tests of exploratory behaviour is not complete without considering the test situation itself: the characteristics of the techniques and apparatus; the testing environment; and the procedural details [88]. All such factors and details should be considered during the planning phase and taken into account when designing the experiments.

First of all, housing and husbandry conditions play a critical role [89]. For instance, single- or group-housing, open or individually-ventilated cages, nesting material all strongly influence the behavioural phenotype [9092]. There is a great deal of evidence that the frequency and method of handling can also influence the behaviour and underlying physiology of experimental animals [93-99]. There is even evidence that the sex of the experimenter can affect the stress response in mice [100] although the systematic follow-up studies to confirm that finding are lacking. Historically, many experiments applied only a single test procedure to study individual animals. However, both the development of genetically-modified mice and the increased attention to reducing unnecessary use of animals have led to the recommended use of test batteries for behavioural phenotyping [101]. 
Although reducing the number of animals used, this approach gives rise to the problem that animals' history of previous testing (including the order of tests) can affect the experimental outcome [102-106]. In general, if testing involves a battery of several tests, the series should start with the assessment of behavioural domains that are more influenced by repetitive exposures, handling and other manipulations. In other words, the natural exploratory drive is studied first, using conventional approach-avoidance tests (e.g., open field, elevated plus (or zero) maze, light-dark box), followed by testing the domains that are thought to be less vulnerable to extensive handling (e.g., motor abilities) or those that even require it (e.g., adaptation to human-animal interaction, which is needed for performing experiments that test cognitive performance).

Additional factors that can influence the behaviour in OFT (and other behavioural experiments) and need to be accounted for at the stage of planning and design are outlined below. Importantly, all these details should be explicitly reported in publications arising from the work because they are critical for objective appraisal of the work, especially when comparing the results with those from other studies.

As well as factors, such as the design and construction of the arena and its illumination, which are discussed in detail (below), several other factors can affect the behaviour of animals in an Open Field

These could include treatment naïve animals, positive controls and negative controls, which could comprise sham-lesioned or vehicle/saline-injected animals.

- The presence of experimenter [100, 108, 109]. It is strongly recommended that the experimenter should be as far away as possible from the animal during the experiment (behind the curtains or even in the neighbouring room). Odours in the room (e.g. perfumes) should be avoided as much as possible, by the use of protective clothing, at least.

- The circadian time of testing. Mice and rats are nocturnal animals: because their activity is naturally higher during dark period, it would be preferable to test the animals during their active period by applying reversed or shifted light-dark cycle. Unfortunately, this is often not possible. In fact, testing either during dark or light period has shown that even if discrimination of strain differences can be better during the dark period, some measures are not affected at all and spatial novelty is equally arousing regardless of circadian phase [110-113]. Nevertheless, circadian rhythms should be taken into account and testing should be carried out approximately at the same time each day, or a randomized block design applied whereby the time of testing is taken into account in the experimental design and statistical analysis of the results [114-116]. On the other hand, it is possible that the experimental intervention causes a phase-shift in the animals' circadian rhythm, in which case, testing at the same time of day could produce a misleading snap-shot of the behavioural 
phenotype [117]. Also, assessment of treatment-induced changes animals' spontaneous locomotor activity should not be based solely on its evaluation in the OFT and needs confirmation by monitoring the animals' activity in the home cage [118].

- Test duration - longer testing may reveal behavioural patterns (habituation, sensitization), which can

\subsection{STANDARDIZATION AND REPRODUCIBILITY}

How to deal with the long list of factors that may confound the outcome of any behavioural experiment?

11 For many years, there has been considerable debate over the benefits versus the drawbacks of rigorous standardization of experimental procedures: in particular, how standardization might improve reproducibility [121-126]. However, a seminal study by Crabbe, Wahlsten and Dudek drew attention to the problem that extensive standardization of the equipment and methods rendered a high possibility for idiosyncratic findings [127]. This is because findings that emerge only under stringently controlled experimental conditions are unlikely to generalise to the animals' real world or be of translational significance. There may be many factors that underlie discrepant results, when comparing findings from studies within, or between laboratories, but there are several studies showing that the person carrying out the experiments may be a major source of variability [109]. On the other hand, that same study also showed the importance of detailed reporting of methods and scrutiny of the data analysis - in line with factors that the ARRIVE guidelines currently promote [128].

Scientific validity of pre-clinical research and animal experiments should built on three pillars - construct validity, internal validity and external validity [129]. Internal validity rests on items listed in the Essential 10 of ARRIVE guidelines (see below) and, in principle, ensures the quality of study conduct, its reliability and consistency of the procedures. However, external validity should be achieved either by controlled and systematic heterogenization of study conditions (e.g. differential housing) by using a randomised block design or by replication of any findings in multi-laboratory studies $[116,123,130]$.

To that end, the core facilities are major instruments for standardizing both the environment and the procedures for behavioural assessment [131-134]. In addition to validating and keeping a record of key test conditions, control experiments (to provide the reference baseline and to ensure that the data are of high quality) should be performed $[133,135]$. Most importantly, staff working in the core facilities should be able and willing to support and advise researchers who may not have the necessary expertise with the methods they plan to use. 


\subsection{RESOURCES FOR RIGOR AND REPRODUCIBILITY}

Unfortunately, several surveys have found a serious lack of rigor in respect of the quality of reporting of important details that can affect results of the experiment, such as those discussed above $[136,137]$. The ARRIVE guidelines were published in 2010 [138] in order to help resolve this problem and improve the reporting of animal research. However, despite endorsement of the guidelines by hundreds of journals, ARRIVE did not have the expected beneficial effect on the quality of research publications [139].

11 As a further remedy, a revised version of the guidelines (ARRIVE 2.0) was published [140], together with extensive accompanying documentation, which explained and elaborated on the revisions [141]. The major change in ARRIVE 2.0 is a categorisation of items that should be included in research reports into two sets: an 'Essential 10' and a 'Recommended'. The Essential 10 comprises many items that are regarded as fundamental to the basics of the experimental design, whereas the Recommended list includes factors that might not be relevant for all types of experiments using animals. Conversely, it is notable that ARRIVE 2.0 has placed many of the items, discussed above, under the 'Recommended' category, but it is important to note that this information should be regarded as mandatory when reporting behavioural research.

There are several other excellent resources that can help deal with all these variables when planning experiments using laboratory animals $[142,143]$, all of which similarly consider the minimum requirements for reporting the in vivo research [144]. Another valuable handbook, covering what is known about the genetic underpinnings of naturally-occurring, non-pathological, individual differences in behaviour, can be highly recommended for researchers who are interested in mice and their behaviour [145] as can another in-depth book, which focuses on many practical aspects of mouse behavioural testing [58]. The legal and ethical aspects of animal experimentation have also been recently reviewed by [146]. Researchers might also find it helpful to consult the Experimental Design Assistant (EDA, available at https://www.nc3rs.org.uk/experimental-design-assistant-eda) - a novel tool bringing together machinereadable flow diagrams and computer-based logical reasoning to assist with the generation of robust and reproducible designs for animal experiments [147]. The contents of all these guidelines serve to emphasize how, even with a test as apparently simple as the OFT, the preparation of the procedure and interpretation of the findings requires full attention to every detail, including the full life-history of the animals, which can affect the outcome of behavioural testing. 
1 It should be recognised that the success of basic, pre-clinical studies and hope for translating the outcomes to clinical research is not possible if the challenges in the design and reporting of animal experiments are not addressed fully and satisfactorily. In this context, an excellent comprehensive review about a wide range of factors that could affect behavioural phenotyping, written in 2010, posed an apposite question in the title: "How many ways can mouse behavioural experiments go wrong?" [148].

\section{MATERIALS}

- Recording equipment and setup (see Figure 2)

These days, tracking and recording of animals' movements is usually done either by infrared photocells or video-tracking systems. If infrared sensors are being used, they should be placed at a height that is appropriate for the size of the test animals. Infrared detectors are sometimes arranged in two levels, in which case the lower array of infrared sensors detects the ambulation and the second, higher level records rearing events (vertical activity).

Video-tracking with a top-down camera requires a good contrast between the test subject and the background [149]. Infrared lights with IR-sensitive camera can be used for enhancing the detection. Additional equipment for refining commercially available systems is available for improving the analysis of video-tracking [150-152] and a number of open-source software platforms for tracking the animals are now available [153-156].

Both systems calculate and store the XY-coordinates of the centre point (geometric centre) of an animal and, based on this information and calibration, its movement is calculated. On the basis of the shape, size and mobility of the detected image, it may be possible to count other behaviours, also: e.g., rearing behaviour or freezing. In addition, the tracking systems may have a feature for manual scoring, meaning that some actions can be recorded during live-tracking or from a video file.

\section{- Physical specifications of the OF}

Larger arenas with a dark floor and low light intensity promote locomotion and exploration [93, 113, 157160]. The early open field arenas were usually circular, but this is less common nowadays, probably because square or rectangular arenas are easier to construct. However, square arenas suffer the problem that animals will prefer the corners of the arena and are likely to show less exploratory activity in other regions of the arena. The walls should be high enough to prevent animals jumping out of the arena. The minimum floor area for mouse is $30 \times 30 \mathrm{~cm}$, for rat $50 \times 50 \mathrm{~cm}$, but a larger arenas are recommended as they will be more sensitive for detecting avoidance of the central region. For video-tracking, the recommended 
1 background (floor) is ideally of grey, non-reflective material, so that there is no light glare at floor level and both albino and pigmented animals can readily be detected.

- Environment-illumination, ambient noise

It is essential that the intensity of illumination is the same in all regions of the arena and that this is checked at floor level, where the animal will be. This can be difficult to achieve with square arenas, especially if they are constructed from shiny, reflective material (e.g. black Perspex). Also, square arenas are vulnerable to producing shadows in the corners, which will not only affect the behaviour of the animals but can pose problem for accurate detection of the animals by video-tracking. The intensity of the illumination can be modified, empirically, according to the protocol (brighter light is generally aversive and can reduce exploratory activity) and so the light intensity at the floor level should be always reported.

Ambient noise at normal levels $(<85 \mathrm{~dB})$ has not been shown to influence the behaviour critically $[88,161]$. However, the environment for behavioural experiments should be protected against major interruptions (e.g. sudden noise bursts, vibration or accidental disturbance by unrelated people).

\section{METHODS}

Several step-by-step protocols for the OFT have been published $[162,163]$. Despite that, the range of procedures in the literature shows that there is no universally accepted protocol. Our aim here is not to prescribe a preferred procedure: rather, we strongly emphasize the importance of attention to details of all the variables discussed in the previous sections that can affect animals' behaviour in the OFT. We argue that every aspect of an animal's biology, life history and environment will affect to the results of the experiment. Therefore, a good knowledge and understanding of laboratory animal science, behavioural neuroscience and experimental design are mandatory elements of training before starting with behavioural testing [164]. However, based on the hypothesis and study questions, standard operating procedures should be written and followed, with all changes to those procedures documented fully and published transparently.

- Prepare the animals and protocols - pay attention to identification, blinding, randomization (the order of testing, counterbalancing the groups between arenas if more than one arena is used).

- If automated recording is used (video-tracking or infrared detection) then calibration and proper connection of hardware must be verified routinely. 
- After taking the animals from the colony room to the test area, give them time to adapt (at least 30 min between transfer and start of test).

- Place the animal in the arena and check that the recording has started. Placement of the animal in either the corner or centre of the arena can influence the resulting behaviour. When placed in the centre, some animals freeze before starting to move. One can measure the latency to move away from the centre as 'escape performance'. When placed in the corner or close to the wall, the animals will most likely start moving along the wall (thigmotaxis) and it will be possible to measure the latency to enter the centre (exposed) area.

- Monitor the animals and the system (remotely) throughout the experiment even if the recording is fully automated.

- After completing the trial remove the animal from the arena and count the number of fecal boli. The latter variable may be indicative of emotionality (if the test duration was short, up to 10 minutes), but it can depend also on other factors (e.g. diet, time elapsed since last eating).

- Clean the arena - different detergents have been used, but also ethanol (5-70\%) or water [165].

- At the end of the test, do not return the tested animal to the home-cage (i.e. cage-mates) until all the animals from the same cage have been tested. It may be important to check for the order effects on behaviour (possible differences between the first and last animal tested from the same cage). This can be achieved by incorporating each animal's position in the sequence of tests as a factor in the statistical analysis.

\section{Results}

These depend largely on the biological and environmental factors. Figure 3 shows representative tracks for different inbred mouse strains in the (square) open field. Proper use of positive and negative controls and strains with known behavioural characteristics will help to confirm that the results are reliable.

The following data are usually collected for analysis: total distance moved; proportion of distance and/or time in the centre area (reference could be around $10-25 \%$ of total); number of rearings (distinguishing those that are supported by contact with the wall from those that are unsupported, in the centre of the arena); speed of movement in periphery and centre; time resting or not moving; any ethological parameters presented previously. 
2 Data analysis is usually done by Analysis of Variance between the groups (with treatment or genotype as a

3 between subjects' factor, for instance). However, the results from the test can be split into time-bins (2-5

4 min, depending on the total duration) for analysis by repeated measures ANOVA to explore whether the

5 influence of the factor(s) of interest (e.g., treatment or genotype) on animals' behaviour(s) change with

6 time in the arena. However, for a comprehensive interpretation of the data it is important to keep in mind

7 that the behaviours are not independent of each other and so factorial ANOVA should be used only when

8 justified by a multivariate analysis, which should be carried out first (see, for example [166]).

Further factors to consider in the experimental design and data analysis

11 - Biological variables: e.g., species, strain, sex, age (discussed above).

12 - Environmental variables: e.g., handling, housing and experimental conditions, testing history 13 (discussed above).

15 To summarize, we fully agree with a recent viewpoint by Lisa Genzel: "In sum, behaviour is not a simple tool 16 or measurement such as measuring the activity of individual neurons or a brain area. Instead, we should 17 always keep in mind that behaviour is the outcome of many different factors. Thus, for one, we should be 18 aware of these factors, keep track of them, and, ideally, control for them as best as we can." [164].

19 Moreover, the behaviour of an animal in any test situation should be described in terms of exactly what it is 20 without unjustified anthropomorphic interpretation $[167,168]$.

\section{ACKNOWLEDGEMENTS}

23 Vootele Voikar is supported by Jane and Aatos Erkko Foundation. 
3 Figure 1. The number of publications per year revealed in PubMed search (run on January 12, 2021) with

4 the following key-words: "open field", "anxiety", "rat OR rats", "mouse OR mice". Note the prevalence of

5 "rat" until mid-1990's and the sharp increase for "mouse" since then (especially for "anxiety").

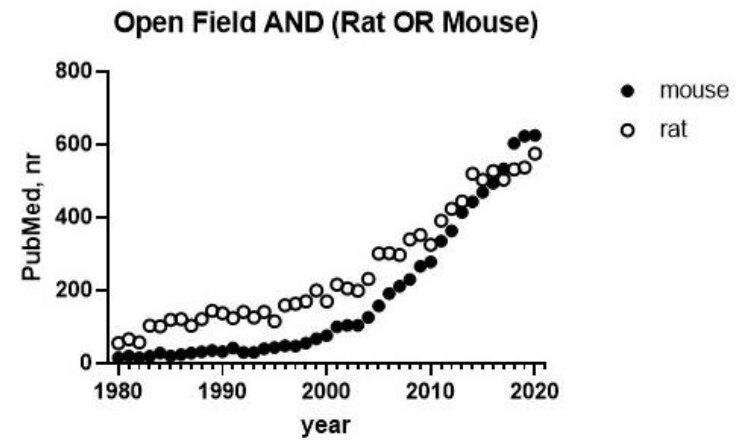

6

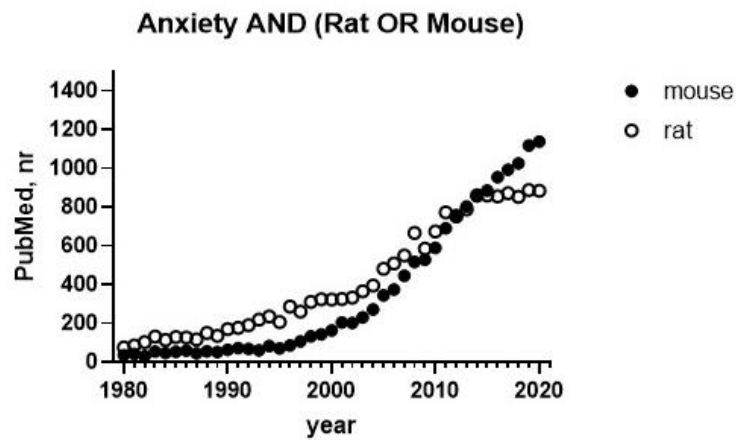

Figure 1.

Figure 2. Two configurations of Open Field setup at the Neuroscience Center, University of Helsinki. Eight arenas (30x30 cm floor) are surrounded by infrared beam arrays at two heights, for detecting horizontal (locomotor) and vertical (rearing) activity (Med Associates, St. Albans, VT).

Another setup consists of four custom-built open field arenas $(50 \times 50 \mathrm{~cm}$ floor) for use with a video-tracking system (Ethovision, Noldus Information Technology, Wageningen, The Netherlands).
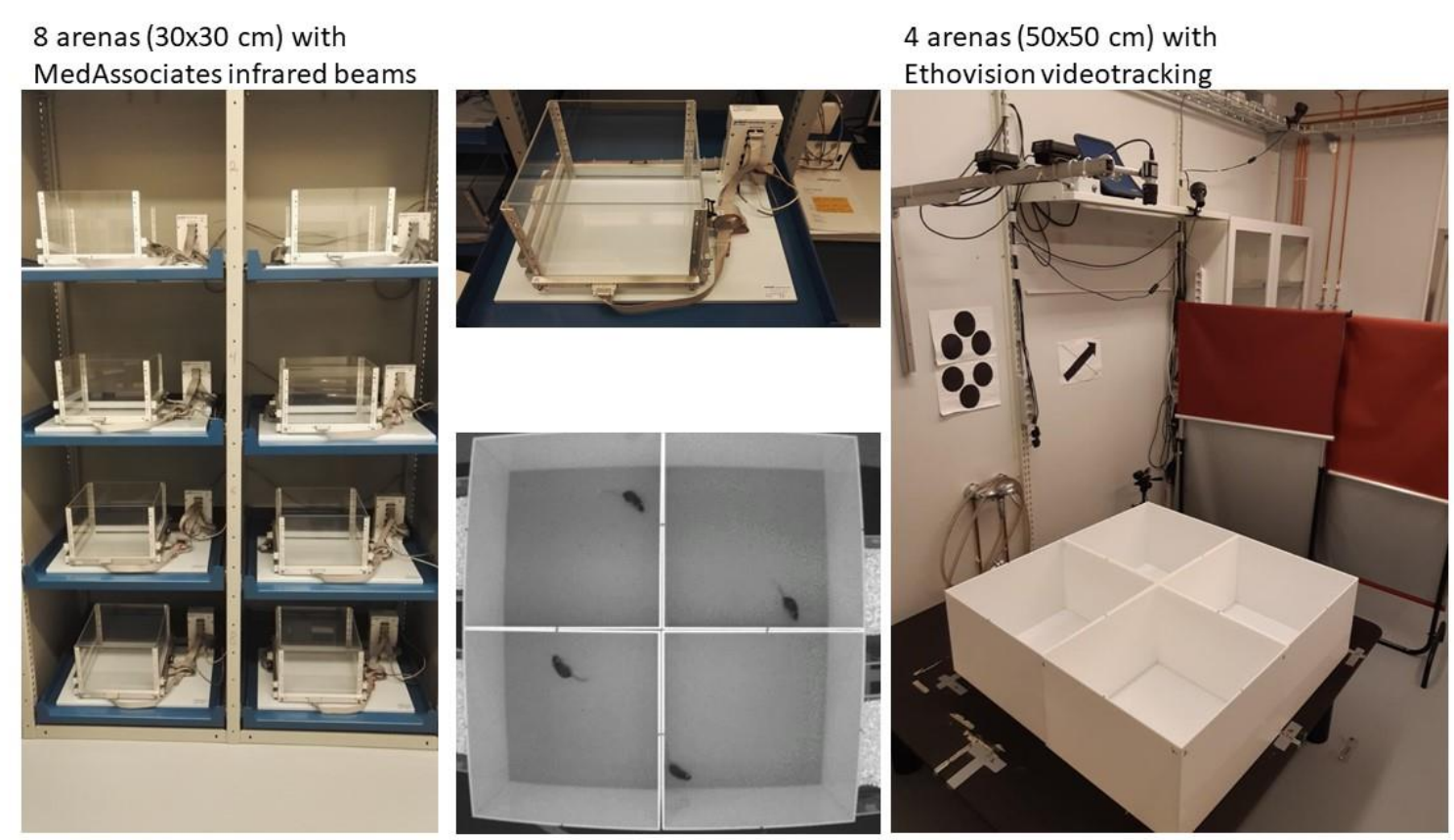

Figure 2. 
1 Figure 3. The typical patterns of activity displayed by three commonly used inbred mouse strains, measured 2 either by using infrared sensors or video-tracking, in arenas of different sizes. Note the difference between 3 the strains in the density of the tracks in the centre of the arena (C57BL/6 > DBA/2 > 129S2). The data are 4 unpublished and derive from pilot experiments carried out at the University of Helsinki (a discussion on 5 strain and equipment differences can be found in Ref. 160, for example).

6

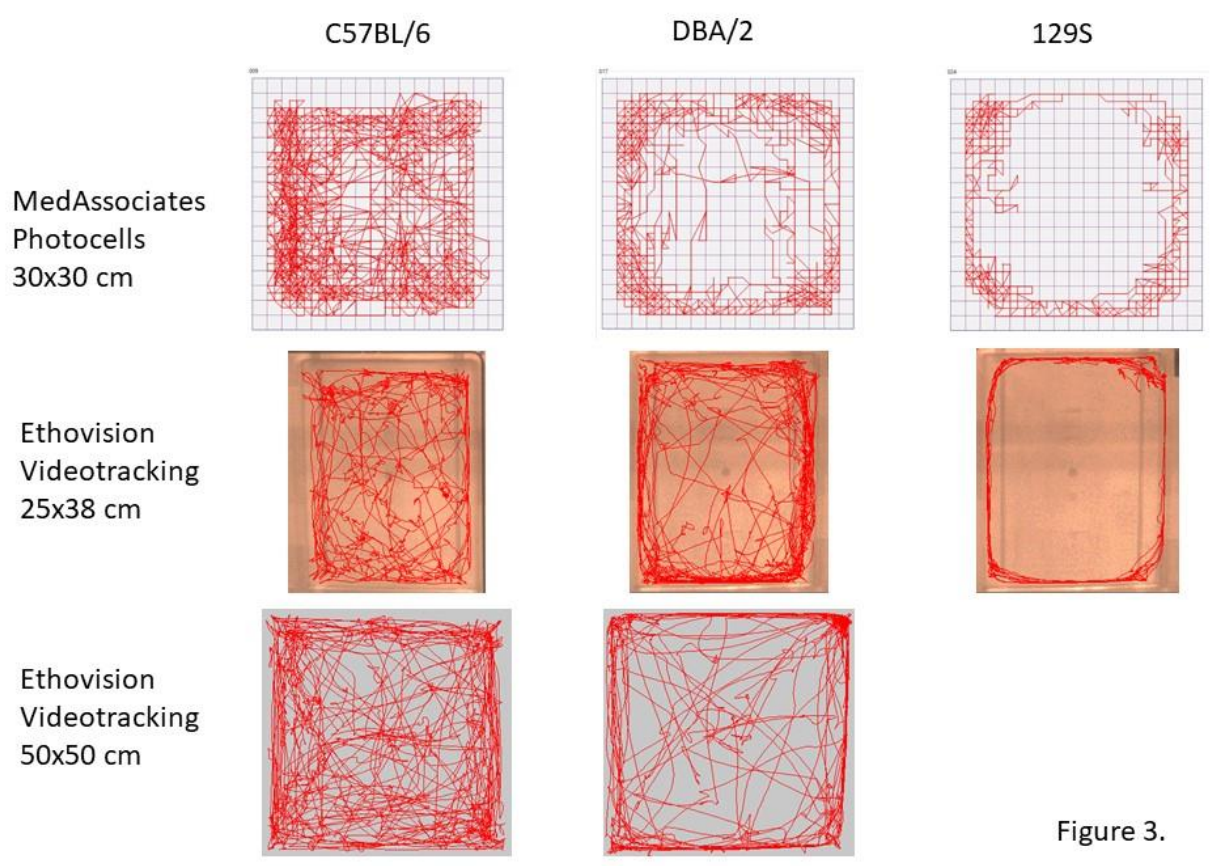


3 1. Hall, C.S., Emotional behaviour in the rat: I. Defecation and urination as measures of

2. Hall, C.S., Emotional behavior in the rat. III. The relationship between emotionality and ambulatory activity. Journal of Comparative Psychology, 1936. 22(3): p. 345-352.

5. Broadhurst, P.L., Determinants of emotionality in the rat: II. Antecedent factors. British

8. Blizard, D.A. and N. Adams, The Maudsley Reactive and Nonreactive strains: a new

9. McQuade, R. and S.C. Stanford, Differences in central noradrenergic and behavioural

10. Royce, J.R., On the construct validity of open-field measures. Psychol Bull, 1977. 84(6): p. 1098-1106.

12. Archer, J., Tests for emotionality in rats and mice: A review. Animal Behaviour, 1973.

13. Denenberg, V.H., Open-field behavior in the rat: what does it mean? Ann N Y Acad Sci, 
1 16. Crawley, J.N., Behavioral phenotyping of transgenic and knockout mice: experimental behavioral tests. Brain Res, 1999. 835(1): p. 18-26.

17. Hanell, A. and N. Marklund, Structured evaluation of rodent behavioral tests used in drug discovery research. Front Behav Neurosci, 2014. 8: p. 252.

18. Crawley, J.N., What's Wrong With My Mouse? Behavioral Phenotyping of Transgenic and Knockout Mice. 2000, New York: Wiley-Liss.

19. Stanford, S.C., The Open Field Test: reinventing the wheel. J Psychopharmacol, 2007. 21(2): p. 134-135.

20. Stanford, S.C., Open fields (unlike wheels) can be any shape but still miss the target. J Psychopharmacol, 2007. 21(2): p. 144.

21. Blizard, D.A., et al., Test standardization in behavioural neuroscience: a response to Stanford. J Psychopharmacol, 2007. 21(2): p. 136-9.

22. Rodgers, R.J., More haste, considerably less speed. J Psychopharmacol, 2007. 21(2): p. 1413.

23. Fonio, E., I. Golani, and Y. Benjamini, Measuring behavior of animal models: faults and remedies. Nat Methods, 2012. 9(12): p. 1167-70.

24. Whishaw, I.Q., et al., Accelerated nervous system development contributes to behavioral efficiency in the laboratory mouse: a behavioral review and theoretical proposal. Dev Psychobiol, 2001. 39(3): p. 151-170.

25. Whishaw, I.Q. and J.A. Tomie, Of mice and mazes: similarities between mice and rats on dry land but not water mazes. Physiol Behav, 1996. 60(5): p. 1191-1197.

26. Whishaw, I.Q., A comparison of rats and mice in a swimming pool place task and matching to place task: some surprising differences. Physiol Behav, 1995. 58(4): p. 687-693.

27. O'Leary, T.P., R.K. Gunn, and R.E. Brown, What are We Measuring When We Test Strain Differences in Anxiety in Mice? Behav Genet, 2013. 43(1): p. 34-50.

28. Takahashi, A., et al., Multivariate analysis of temporal descriptions of open-field behavior in wild-derived mouse strains. Behav Genet, 2006. 36(5): p. 763-74.

29. Drai, D., et al., Rats and mice share common ethologically relevant parameters of exploratory behavior. Behav Brain Res, 2001. 125(1-2): p. 133-140.

30. Suarez, S.D. and G.G. Gallup, An ethological analysis of open-field behavior in rats and mice. Learning and Motivation, 1981. 12(3): p. 342-363.

31. Welker, W.I., "Free” versus "Forced” Exploration of a Novel Situation by Rats. Psychological Reports, 1957. 3(1): p. 95-108. 
32. Fonio, E., Y. Benjamini, and I. Golani, Short and long term measures of anxiety exhibit opposite results. PLoS ONE, 2012. 7(10): p. e48414.

33. Bolivar, V.J., et al., Habituation of activity in an open field: A survey of inbred strains and F1 hybrids. Behav Genet, 2000. 30(4): p. 285-293.

34. Leussis, M.P. and V.J. Bolivar, Habituation in rodents: A review of behavior, neurobiology, and genetics. Neurosci Biobehav Rev, 2006. 30(7): p. 1045-1064.

35. Bolivar, V.J., Intrasession and intersession habituation in mice: From inbred strain variability to linkage analysis. Neurobiology of Learning and Memory, 2009. 92(2): p. 206214.

36. Lipkind, D., et al., New replicable anxiety-related measures of wall vs center behavior of mice in the open field. J Appl Physiol, 2004. 97(1): p. 347-59.

37. Angrini, M., J.C. Leslie, and R.A. Shephard, Effects of propranolol, buspirone, pCPA, reserpine, and chlordiazepoxide on open-field behavior. Pharmacol Biochem Behav, 1998. 59(2): p. 387-97.

38. Salmon, P. and S.C. Stanford, Beta-adrenoceptor binding correlates with behaviour of rats in the open field. Psychopharmacology (Berl), 1989. 98(3): p. 412-6.

39. Lindquist, M.P. and K.G. Götestam, Open-field behavior after intravenous amphetamine analogues in rats. Psychopharmacology (Berl), 1977. 55(2): p. 129-33.

40. Zimcikova, E., et al., Behavioral effects of antiepileptic drugs in rats: Are the effects on mood and behavior detectable in open-field test? Seizure, 2017. 52: p. 35-40.

41. Thompson, S.M., L.E. Berkowitz, and B.J. Clark, Behavioral and Neural Subsystems of Rodent Exploration. Learn Motiv, 2018. 61: p. 3-15.

42. Eilam, D. and I. Golani, Home base behavior of rats (Rattus norvegicus) exploring a novel environment. Behavioural Brain Research, 1989. 34(3): p. 199-211.

43. van Abeelen, J.H.F., Genetics of rearing behavior in mice. Behavior Genetics, 1970. 1(1): p. 71-76.

44. van Abeelen, J.H.F., P.H.W. van der Kroon, and M.F.J. Bekkers, Mice selected for rearing behavior: Some physiological variables. Behavior Genetics, 1973. 3(1): p. 85-90.

45. Lever, C., S. Burton, and J. O'Keefe, Rearing on hind legs, environmental novelty, and the hippocampal formation. Rev Neurosci, 2006. 17(1-2): p. 111-33.

46. Sturman, O., P.L. Germain, and J. Bohacek, Exploratory rearing: a context- and stresssensitive behavior recorded in the open-field test. Stress, 2018: p. 1-10.

47. Delprato, A., et al., QTL and systems genetics analysis of mouse grooming and behavioral responses to novelty in an open field. Genes Brain Behav, 2017. 16(8): p. 790-799. 
48. Estanislau, C., Cues to the usefulness of grooming behavior in the evaluation of anxiety in the elevated plus-maze. Psychology \& Neuroscience, 2012. 5: p. 105-112.

49. Fernández-Teruel, A. and C. Estanislau, Meanings of self-grooming depend on an inverted U-shaped function with aversiveness. Nature Reviews Neuroscience, 2016. 17(9): p. 591591.

50. Kalueff, A.V., et al., Neurobiology of rodent self-grooming and its value for translational neuroscience. Nat Rev Neurosci, 2016. 17(1): p. 45-59.

51. van den Boom, B.J.G., et al., Automated classification of self-grooming in mice using opensource software. J Neurosci Methods, 2017. 289: p. 48-56.

52. Spruijt, B.M., J.A. van Hooff, and W.H. Gispen, Ethology and neurobiology of grooming behavior. Physiol Rev, 1992. 72(3): p. 825-852.

53. Lester, D., The effect of fear and anxiety on exploration and curiosity: Toward a theory of exploration. Journal of General Psychology, 1968. 79(1): p. 105-120.

54. Barnett, S. and P. Cowan, Activity, Exploration, Curiosity and Fear: An Ethological Study. Interdisciplinary Science Reviews, 1976. 1: p. 43-62.

55. van Abeelen, J.H.F., Mouse mutants studied by means of ethological methods. I. Ethogram. Genetica, 1964. 34(1): p. 79-94.

56. Grant, E.C. and J.H. Mackintosh, A Comparison of the Social Postures of Some Common Laboratory Rodents. Behaviour, 1963. 21(3/4): p. 246-259.

57. Irwin, S., Comprehensive observational assessment: Ia. A systematic, quantitative procedure for assessing the behavioral and physiologic state of the mouse.

Psychopharmacologia, 1968. 13(3): p. 222-257.

58. Wahlsten, D., Mouse Behavioral Testing. How to use mice in behavioral neuroscience. 2011: Academic Press.

59. Pereira, T.D., J.W. Shaevitz, and M. Murthy, Quantifying behavior to understand the brain. Nat Neurosci, 2020. 23(12): p. 1537-1549.

60. Mathis, M.W. and A. Mathis, Deep learning tools for the measurement of animal behavior in neuroscience. Current Opinion in Neurobiology, 2020. 60: p. 1-11.

61. Wiltschko, A.B., et al., Revealing the structure of pharmacobehavioral space through motion sequencing. Nat Neurosci, 2020. 23(11): p. 1433-1443.

62. von Ziegler, L., O. Sturman, and J. Bohacek, Big behavior: challenges and opportunities in a new era of deep behavior profiling. Neuropsychopharmacology, 2021. 46(1): p. 33-44. 
1 63. Sturman, O., et al., Deep learning-based behavioral analysis reaches human accuracy and 45(11): p. 1942-1952.

64. Hobson, L., et al., Phenotyping in Mice Using Continuous Home Cage Monitoring and Ultrasonic Vocalization Recordings. Current Protocols in Mouse Biology, 2020. 10(3): p. e80.

65. Zucker, I. and A.K. Beery, Males still dominate animal studies. Nature, 2010. 465(7299): p. 690.

66. Beery, A.K. and I. Zucker, Sex bias in neuroscience and biomedical research. Neurosci Biobehav Rev, 2011. 35(3): p. 565-72.

67. Gray, J.A., Sex differences in emotional behaviour in mammals including man: Endocrine bases. Acta Psychologica, 1971. 35(1): p. 29-46.

68. Johnston, A.L. and S.E. File, Sex differences in animal tests of anxiety. Physiology \& Behavior, 1991. 49(2): p. 245-250.

69. Gray, J.A., Emotionality in male and female rodents: a reply to Archer. Br J Psychol, 1979. 70(3): p. 425-40.

70. Palanza, P., Animal models of anxiety and depression: how are females different? Neurosci Biobehav Rev, 2001. 25(3): p. 219-233.

71. Voikar, V., et al., Strain and gender differences in the behavior of mouse lines commonly used in transgenic studies. Physiol Behav, 2001. 72(1-2): p. 271-281.

72. Mogil, J.S., et al., Sex differences in thermal nociception and morphine antinociception in rodents depend on genotype. Neurosci Biobehav Rev, 2000. 24(3): p. 375-389.

73. Prendergast, B.J., K.G. Onishi, and I. Zucker, Female mice liberated for inclusion in neuroscience and biomedical research. Neurosci Biobehav Rev, 2014. 40: p. 1-5.

74. Karp, N.A. and N. Reavey, Sex bias in preclinical research and an exploration of how to change the status quo. Br J Pharmacol, 2019. 176(21): p. 4107-4118.

75. Fritz, A.K., I. Amrein, and D.P. Wolfer, Similar reliability and equivalent performance of female and male mice in the open field and water-maze place navigation task. Am J Med Genet C Semin Med Genet, 2017. 175(3): p. 380-391.

76. Clayton, J.A., Applying the new SABV (sex as a biological variable) policy to research and clinical care. Physiol Behav, 2018. 187: p. 2-5.

77. Clayton, J.A. and F.S. Collins, Policy: NIH to balance sex in cell and animal studies. Nature, 2014. 509(7500): p. 282-3.

78. Mogil, J.S., Perspective: Equality need not be painful. Nature, 2016. 535(7611): p. S7-S7. 
79. Wittchen, H.U., et al., The size and burden of mental disorders and other disorders of the

80. Miller, L.R., et al., Considering sex as a biological variable in preclinical research. Faseb j, 2017. 31(1): p. 29-34.

81. Docherty, J.R., et al., Sex: A change in our guidelines to authors to ensure that this is no longer an ignored experimental variable. British Journal of Pharmacology, 2019. 176(21): p. 4081-4086.

82. Tuttle, A.H., et al., Comparing phenotypic variation between inbred and outbred mice. Nature Methods, 2018. 15(12): p. 994-996.

83. Festing, M.F., Warning: the use of heterogeneous mice may seriously damage your research. Neurobiol Aging, 1999. 20(2): p. 237-44; discussion 245-6.

84. Festing, M.F., Evidence should trump intuition by preferring inbred strains to outbred stocks in preclinical research. ILAR J, 2014. 55(3): p. 399-404.

85. Logue, S.F., et al., Assessment of locomotor activity, acoustic and tactile startle, and prepulse inhibition of startle in inbred mouse strains and F1 hybrids: implications of genetic background for single gene and quantitative trait loci analyses. Neuroscience, 1997. 80(4): p. 1075-1086.

86. Crawley, J.N., et al., Behavioral phenotypes of inbred mouse strains: implications and recommendations for molecular studies. Psychopharmacology (Berl), 1997. 132(2): p. 107124.

87. Holmes, A., et al., Abnormal anxiety-related behavior in serotonin transporter null mutant mice: the influence of genetic background. Genes Brain Behav, 2003. 2(6): p. 365-380.

88. Walsh, R.N. and R.A. Cummins, The Open-Field Test: a critical review. Psychol Bull, 1976. 83(3): p. 482-504.

89. Roubertoux, P.L., Maintaining Mice for Neurobehavioral Examination. Neuromethods, 2015. 100: p. 319-333.

90. Voikar, V., et al., Long-term individual housing in C57BL/6J and DBA/2 mice: assessment of behavioral consequences. Genes Brain Behav, 2005. 4(4): p. 240-252.

91. Kulesskaya, N., H. Rauvala, and V. Voikar, Evaluation of Social and Physical Enrichment in Modulation of Behavioural Phenotype in C57BL/6J Female Mice. PLoS ONE, 2011. 6(9): p. e24755.

92. Ahlgren, J. and V. Voikar, Housing mice in the individually ventilated or open cages-Does it matter for behavioral phenotype? Genes Brain Behav, 2019. 18(7): p. e12564. 
93. Igarashi, E. and S. Takeshita, Effects of illumination and handling upon rat open field activity. Physiology \& Behavior, 1995. 57(4): p. 699-703.

94. Deacon, R.M., Housing, husbandry and handling of rodents for behavioral experiments. Nat

95. Hurst, J.L. and R.S. West, Taming anxiety in laboratory mice. Nat Methods, 2010. 7: p. 825-

96. Andrews, N. and S.E. File, Handling history of rats modifies behavioural effects of drugs in

97. File, S.E. and E. Fluck, Handling alters habituation and response to stimulus change in the

98. Bohlen, M., et al., Experimenter effects on behavioral test scores of eight inbred mouse strains under the influence of ethanol. Behav Brain Res, 2014. 272: p. 46-54.

99. Stanford, C., M. Fillenz, and E. Ryan, The effect of repeated mild stress on cerebral cortical adrenoceptors and noradrenaline synthesis in the rat. Neurosci Lett, 1984. 45(2): p. 163-7.

100. Sorge, R.E., et al., Olfactory exposure to males, including men, causes stress and related analgesia in rodents. Nat Methods, 2014. 11(6): p. 629-32.

101. Crawley, J.N. and R. Paylor, A proposed test battery and constellations of specific behavioral paradigms to investigate the behavioral phenotypes of transgenic and knockout mice. Horm Behav, 1997. 31(3): p. 197-211.

102. McIlwain, K.L., et al., The use of behavioral test batteries: Effects of training history. Physiol Behav, 2001. 73(5): p. 705-717.

103. Blokland, A., et al., The use of a test battery assessing affective behavior in rats: Order effects. Behavioural Brain Research, 2012. 228(1): p. 16-21.

104. Bouwknecht, J.A., et al., Effects of repeated testing in two inbred strains on flesinoxan doseresponse curves in three mouse models for anxiety. Eur J Pharmacol, 2004. 494(1): p. 35-44.

105. Paylor, R., et al., The use of behavioral test batteries, II: Effect of test interval. Physiol Behav, 2006. 87(1): p. 95-102.

106. Voikar, V., E. Vasar, and H. Rauvala, Behavioral alterations induced by repeated testing in C57BL/6J and 129S2/Sv mice: implications for phenotyping screens. Genes Brain Behav, 2004. 3(1): p. 27-38.

107. Lapin, I.P., Only controls: effect of handling, sham injection, and intraperitoneal injection of saline on behavior of mice in an elevated plus-maze. J Pharmacol Toxicol Methods, 1995. 34(2): p. 73-77. 
1 108. Hughes, C.W., Observer influence on automated open field activity. Physiology \& Behavior, 1978. 20(4): p. 481-485.

109. Chesler, E.J., et al., Identification and ranking of genetic and laboratory environment factors influencing a behavioral trait, thermal nociception, via computational analysis of a large data archive. Neurosci Biobehav Rev, 2002. 26(8): p. 907-923.

110. Hossain, S.M., B.K. Wong, and E.M. Simpson, The dark phase improves genetic discrimination for some high throughput mouse behavioral phenotyping. Genes Brain Behav, 2004. 3(3): p. 167-177.

111. Yang, M., M.D. Weber, and J.N. Crawley, Light phase testing of social behaviors: not a problem. Front Neurosci, 2008. 2(2): p. 186-91.

112. Beeler, J.A., B. Prendergast, and X. Zhuang, Low amplitude entrainment of mice and the impact of circadian phase on behavior tests. Physiology \& Behavior, 2006. 87(5): p. 870880 .

113. Valentinuzzi, V.S., et al., Locomotor response to an open field during C57BL/6J active and inactive phases: differences dependent on conditions of illumination. Physiol Behav, 2000. 69(3): p. 269-275.

114. Bodden, C., et al., Heterogenising study samples across testing time improves reproducibility of behavioural data. Scientific Reports, 2019. 9(1): p. 8247.

115. Festing, M.F., The scope for improving the design of laboratory animal experiments. Lab Anim, 1992. 26(4): p. 256-68.

116. Festing, M.F.W., The "completely randomised" and the "randomised block" are the only experimental designs suitable for widespread use in pre-clinical research. Scientific Reports, 2020. 10(1): p. 17577.

117. Porter, A.J., et al., A lack of functional NK1 receptors explains most, but not all, abnormal behaviours of NK1R-/- mice(1). Genes Brain Behav, 2015. 14(2): p. 189-99.

118. Voikar, V. and S. Gaburro, Three Pillars of Automated Home-Cage Phenotyping of Mice: Novel Findings, Refinement, and Reproducibility Based on Literature and Experience. Frontiers in Behavioral Neuroscience, 2020. 14: p. 193.

119. Henriques-Alves, A.M. and C.M. Queiroz, Ethological Evaluation of the Effects of Social Defeat Stress in Mice: Beyond the Social Interaction Ratio. Front Behav Neurosci, 2016. 9: p. 364.

120. Morello, F., et al., ADHD-like behaviors caused by inactivation of a transcription factor controlling the balance of inhibitory and excitatory neuron development in the mouse anterior brainstem. Translational Psychiatry, 2020. 10(1): p. 357. 
121. Van der Staay, F.J. and T. Steckler, The fallacy of behavioral phenotyping without standardisation. Genes Brain Behav, 2002. 1(1): p. 9-13.

122. van der Staay, F.J., S.S. Arndt, and R.E. Nordquist, The standardization-generalization dilemma: A way out. Genes Brain Behav, 2010. 9(8): p. 849-855.

123. Voelkl, B., et al., Reproducibility of animal research in light of biological variation. Nat Rev Neurosci, 2020. 21(7): p. 384-393.

124. Würbel, H., Behaviour and the standardization fallacy. Nat Genet, 2000. 26(3): p. 263.

125. Kafkafi, N., et al., Reproducibility and replicability of rodent phenotyping in preclinical studies. Neurosci Biobehav Rev, 2018. 87: p. 218-232.

126. Crabbe, J.C., Reproducibility of Experiments with Laboratory Animals: What Should We Do Now? Alcohol Clin Exp Res, 2016. 40(11): p. 2305-2308.

127. Crabbe, J.C., D. Wahlsten, and B.C. Dudek, Genetics of mouse behavior: interactions with laboratory environment. Science, 1999. 284(5420): p. 1670-1672.

128. Wahlsten, D., et al., Different data from different labs: lessons from studies of geneenvironment interaction. J Neurobiol, 2003. 54(1): p. 283-311.

129. Wurbel, H., More than 3Rs: the importance of scientific validity for harm-benefit analysis of animal research. Lab Anim (NY), 2017. 46(4): p. 164-166.

130. Karp, N.A., Reproducible preclinical research-Is embracing variability the answer? PLoS Biol, 2018. 16(3): p. e2005413.

131. Bikovski, L., et al., Lessons, insights and newly developed tools emerging from behavioral phenotyping core facilities. J Neurosci Methods, 2020. 334: p. 108597.

132. Kos-Braun, I.C., B. Gerlach, and C. Pitzer, Science Forum: A survey of research quality in core facilities. eLife, 2020. 9: p. e62212.

133. Voikar, V., Reproducibility of behavioral phenotypes in mouse models - short history with critical and practical notes. Journal for Reproducibility in Neuroscience, 2020. 1(1).

134. Gulinello, M., et al., Rigor and reproducibility in rodent behavioral research. Neurobiol Learn Mem, 2019. 165: p. 106780.

135. Blizard, D.A., et al., Use of a standard strain for external calibration in behavioral phenotyping. Behav Genet, 2005. 35(3): p. 323-32.

136. Kilkenny, C., et al., Survey of the quality of experimental design, statistical analysis and reporting of research using animals. PLoS ONE, 2009. 4(11): p. e7824.

137. Prager, E.M., et al., The importance of reporting housing and husbandry in rat research. Front Behav Neurosci, 2011. 5: p. 38. 
1 138. Kilkenny, C., et al., Improving bioscience research reporting: the ARRIVE guidelines for reporting animal research. PLoS Biol, 2010. 8(6): p. e1000412.

139. Leung, V., et al., ARRIVE has not ARRIVEd: Support for the ARRIVE (Animal Research: Reporting of in vivo Experiments) guidelines does not improve the reporting quality of papers in animal welfare, analgesia or anesthesia. PLOS ONE, 2018. 13(5): p. e0197882.

140. Percie du Sert, N., et al., The ARRIVE guidelines 2.0: Updated guidelines for reporting animal research. PLOS Biology, 2020. 18(7): p. e3000410.

141. Percie du Sert, N., et al., Reporting animal research: Explanation and elaboration for the ARRIVE guidelines 2.0. PLOS Biology, 2020. 18(7): p. e3000411.

142. Smith, A.J., et al., PREPARE: guidelines for planning animal research and testing. Lab Anim, 2018. 52(2): p. 135-141.

143. Guidelines Guiding Principles for Behavioural Laboratory Animal Science. BAP Consensus Guidelines, 2013.

144. Voehringer, P. and J.R. Nicholson, Minimum Information in In Vivo Research. Handb Exp Pharmacol, 2020. 257: p. 197-222.

145. Crusio, W.E., et al., eds. Behavioral Genetics of the Mouse. Volume 1. Genetics of Behavioral Phenotypes. Cambridge Handbooks in Behavioral Genetics. 2013, Cambridge University Press.

146. Guillén, J. and T. Steckler, Good Research Practice: Lessons from Animal Care and Use. Handb Exp Pharmacol, 2020. 257: p. 367-382.

147. Percie du Sert, N., et al., The Experimental Design Assistant. PLoS Biol, 2017. 15(9): p. e2003779.

148. Schellinck, H.M., D.P. Cyr, and R.E. Brown, How Many Ways Can Mouse Behavioral Experiments Go Wrong? Confounding Variables in Mouse Models of Neurodegenerative Diseases and How to Control Them, in Advances in the Study of Behavior, T.J.R. H. Jane Brockmann, Marc Naguib, Katherine E. Wynne-Edwards, John C. Mitani, Leigh W. Simmons, Editor. 2010, Academic Press. p. 255-366.

149. Bailoo, J.D., M.O. Bohlen, and D. Wahlsten, The precision of video and photocell tracking systems and the elimination of tracking errors with infrared backlighting. J Neurosci Methods, 2010. 188(1): p. 45-52.

150. Kafkafi, N., et al., SEE locomotor behavior test discriminates C57BL/6J and DBA/2J mouse inbred strains across laboratories and protocol conditions. Behav Neurosci, 2003. 117(3): p. 464-477. 
151. Wolfer, D.P. and H.P. Lipp, A new computer program for detailed off-line analysis of swimming navigation in the Morris water maze. J Neurosci Methods, 1992. 41(1): p. 65-74.

152. Wolfer, D.P., et al., Extended analysis of path data from mutant mice using the public

153. Pennington, Z.T., et al., ezTrack: An open-source video analysis pipeline for the investigation of animal behavior. Scientific Reports, 2019. 9(1): p. 19979.

154. Aguiar, P., L. Mendonça, and V. Galhardo, OpenControl: A free opensource software for video tracking and automated control of behavioral mazes. Journal of Neuroscience Methods, 2007. 166(1): p. 66-72.

155. Sridhar, V.H., D.G. Roche, and S. Gingins, Tracktor: Image-based automated tracking of animal movement and behaviour. Methods in Ecology and Evolution, 2019. 10(6): p. 815820.

156. Zhang, C., H. Li, and R. Han, An open-source video tracking system for mouse locomotor activity analysis. BMC Research Notes, 2020. 13(1): p. 48.

157. Dixon, L.K. and J.C. DeFries, Effects of illumination on open-field behavior in mice. Journal of Comparative and Physiological Psychology, 1968. 66(3, Pt.1): p. 803-805.

158. McReynolds, W.E., M.W. Weir, and J.C. DeFries, Open-field behavior in mice: Effect of test illumination. Psychonomic Science, 1967. 9(5): p. 277-278.

159. Valle, F.P., Effects of Strain, Sex, and Illumination on Open-Field Behavior of Rats. The American Journal of Psychology, 1970. 83(1): p. 103-111.

160. Kulesskaya, N. and V. Voikar, Assessment of mouse anxiety-like behaviour in the light-dark box and open-field arena: Role of equipment and procedure. Physiol Behav, 2014. 133: p. 30-38.

161. Turner, J.G., et al., Hearing in laboratory animals: strain differences and nonauditory effects of noise. Comp Med, 2005. 55(1): p. 12-23.

162. Kraeuter, A.K., P.C. Guest, and Z. Sarnyai, The Open Field Test for Measuring Locomotor Activity and Anxiety-Like Behavior. Methods Mol Biol, 2019. 1916: p. 99-103.

163. Seibenhener, M.L. and M.C. Wooten, Use of the Open Field Maze to measure locomotor and anxiety-like behavior in mice. J Vis Exp, 2015(96): p. e52434.

164. Genzel, L., How to Control Behavioral Studies for Rodents-Don't Project Human Thoughts onto Them. eneuro, 2021. 8(1): p. ENEURO.0456-20.2021.

165. Hascoet, M., M. Bourin, and B.A. Dhonnchadha, The mouse light-dark paradigm: a review. Prog Neuropsychopharmacol Biol Psychiatry, 2001. 25(1): p. 141-166. 
1 166. Bate, S.T., R.A. Clark, and S.C. Stanford, Using InVivoStat to perform the statistical analysis of experiments. Journal of Psychopharmacology, 2017. 31(6): p. 644-652.

167. Stanford, S.C., Confusing preclinical (predictive) drug screens with animal 'models' of psychiatric disorders, or 'disorder-like' behaviour, is undermining confidence in behavioural neuroscience. J Psychopharmacol, 2017. 31(6): p. 641-643.

168. Stanford, S.C., Some Reasons Why Preclinical Studies of Psychiatric Disorders Fail to Translate: What Can Be Rescued from the Misunderstanding and Misuse of Animal 'Models'? Alternatives to Laboratory Animals, 2020: p. 0261192920939876. 